\title{
Analysis of the Image-Building and Costume Design of Host Group of TV Show
}

\author{
Mengmeng Hou \\ Eastern International Art College \\ Zhengzhou University of Light Industry \\ Zhengzhou, China 451450
}

\begin{abstract}
The host image is the most striking visual symbols in TV programs, the subject and orientation of a program can be reflected by the costume of host from a macro point of view, and the character and connotation of the host can be embodied via his costume from micro point of view. At present, the position of host as bridge connected the TV media and the audience is much more important, which represents the features of program and the media image to some extent. There is the difference of the host group, as a special mode in hostess, in costume design and model design.
\end{abstract}

Keywords-host group; tv program; media; image-building; costume design

\section{INTRODUCTION}

In the alternation time between the old and new media, television, as a inevitable force in the mass media, still carries all kinds of complicated information and fills in every corner of the life of the masses.TV media, after nearly a decade of booming development, has become a very mature industry, but its content is still in the ascendant, it not only make the life of the masses more colorful via the news, entertainment and variety, but also let the audience puts forward higher requirements with more and more professional sight to the quality of TV programs, which has brought great challenge to TV production[1-3].

In these challenges, the costume design is an important part for image-building of the host, because it covers the development concept and orientation of TV and is close to the TV brand pursuit and brand awareness, thus the unique style of costume art for the host is formed[4]. The host group concept raised in 2008 when the "new weekly" issued the best host award to "everyday brothers" hosted Make Progress Everyday of Hunan satellite TV brought a greater challenge to host costume design, host group also stand in the forefront of TV programs like the common host, whose image in words and deeds and looks up represents the image of the style of TV programs at a certain extent, the costume design of each host among the host group is particularly important for the image-building of host group and the whole image of the TV program.

\section{SER VICE OF THE COSTUME Design OF THE HOST FOR IMAGE-BUILDING}

Clothing is a microcosm of the era for the aesthetic, and the costume style of the host group not only should meet the main style of TV programs, but also must be in accordance with the cultural aesthetic of the era in fashion aspects and reflect national cultural connotations. To understand the application of the clothing of the host group in imagebuilding can draw the evolution road map of the clothing of the host group, which is good for predict the future direction of image-building, at the same time, the research combining with the background evolution of the era and the evolution characteristics of the clothing of host group helps to explore the trend of the aesthetic consciousness of people, and the research on the image-building, color and fabric and so on of the costume of the host group helps to further explore the essence of clothing image-building and be better applied to the more excellent TV programs.

\section{A. The Costume Style of Host Group and the Image Orientation of TV Show Are Unified}

The clothing style of host group of any kinds of programs should take the orientation of the image-building of the host group as reference. The connotation of the program can be fully embodied only when the costume style of host group and the image orientation of TV show are unified. Different programs need different clothing styles, which can not be too serious like traditional news programs, too gorgeous like a host of literature and art and too rigorous like a professional program. First deeply understand the program connotation and comprehensively connect the individual characteristic of the host group to unify the clothing style and image-building of host group; such as the Taiwan program University specially designed for the university students explores the different views of the young man via different tests to different students, so the costume design of the three hosts of Jingying Tao, Nadou and A Ken is close to the university students and young man. If the costume of the host group is out of the way of audience expectation for the program orientation, it will have a negative impact on program spread, which likes wrong-tune music is out of the music. The practice has proved that the program can win the audience's approval only when the costume of the host group matches the image-building and meets the demands of audience, the 
clothing design is meaningless if it is not aiming at imagebuilding.

\section{B. Clothing Design Is A Sword to Avoid Image-building Homogeneity}

With the increasing quantity and category of TV programs, the program unavoidably appears homogeneity on the positioning, and if there is no difference on the clothing design and style of the host group, there will be many same TV programs which will not arouse the audience's interest, so advantage of positing the image-building for host group via the clothing design is highlighted. In the specific program types such as the big parts of television news, the orientation of the shows is same, which mainly is the updates for news and the costume design under the positioning shows, rarely see innovative press plate. In contrast, TV shows hosted by host group are generally entertainment programs, which variety on programs is much more than others, so to set up the image of host group has brought large imaging space for clothing design. The service thinking consciously formed via taking costume design of host group as foothold and basing on fully cognitive basis to the positioning of a program of positioning and image-building, indicates the host group must first hint himself is a part of the program image, and the meaning of dress should be in conformity with this. So, under the pre-condition of ensuring the unification to the show orientation, the host group should try his best to pursuit the characteristic style of costume, which shows the cultural pursuit and aesthetic tendency of the program and the imagebuilding of the host group, reduce the fashion sense of the clothing when it is necessary to avoid the homogeneity for image-building.

\section{The Consistency of Costume Design Builds the Continuity of Image-Building}

TV show host image are consistent with program positioning, which needs to keep the continuity of change, clothing design consistency on keeping the continuity is an important driving force. Maintain the consistency of the host dress style does not mean a layer of constant, but have change on the change of rhythm. Generally speaking, the image of host group is stable in the heart of the audience, if the host group blindly pursue unilateral change, then it will cause the loss of the intangible value, and is lack of stability and continuity. At present, the variety shows with the longest establishment time in domestic is the "Happy Camp" of Hunan satellite TV premiered in July 1997, during the continuous host for more than ten years, the clothing style of the host, Jiong He, has always followed the young man's aesthetic trend, although have change, also have a lot of continuity, which will not cut host image and block the condensation of the show style, but brings up the grand unification between the program style and the dress style. Floating dress style in the TV show is difficult to form the audience's recognition degree and consistency for spreading the image of host group. Therefore, to maintain the unity of the clothing style and follow the continuity and stability in the change is an important principle in the clothing design for the host.

\section{THE Distinctiveness OF THE COSTUME DESIGN OF THE HOST GROUP IN IMAGE-BUILDING}

Costume design in the service in image creation at the same time, as the media for the promotion of fashion and clothing design of antenna has been born to all spheres of life, the development of television media makes show host group of dress also occupies the important position. Different from other types of apparel design, host group of dress design needs to consider various factors, program positioning, theme, presiding over the characteristics of the group of individual and group the whole style to clothing design put forward higher requirements. Coupled with the influence of TV programs, the host clothing every button, it means a lot, so the host group of clothing design should be targeted and seriation research, in order to better use of costume design in the characteristics of the host of the clear of image creation, to speed up the development of costume culture.

\section{A. Costume Design of the Host Group for Image-building Orientation}

The aesthetics of costume creation are full of uncertainty and probability, and it is the incarnation of creative activity fusing personal feelings of a designer, costume design of the host focuses on originality like all other art creation. Clothing design is the silent language showing the image modeling of host group, and it includes the individuality of the host group and the orientation of show style. The clothing has distinctive features. It is also possible that orient the costume design via the image of the host group. Such as the host Handian Chen plays the whimsy role in Taiwan variety show "Kangxi Come", which highlights the knowledgeable Kangyong Cai and versatile Xidi Xu, so the costume of Yongkai Cai always adopts the mature style, the one of Xidi Xu is good to show the female charm, while the clothing of Handian Chen often is cross-dressing which is in line with the image orientation of the host group with its exaggerated model.

The host $\mathrm{Na}$ Xie in another show of "Happy Camp" also has individual character with her young, dynamic and natural image, she honestly hosts guests and the audience which very match the and the "happiness" positioning of the program.

\section{B. The Innovation Elements of the Clothing Design Lightens the Image-Building}

From design point of view, the so-called clothing creative design is the combination of artistic creation of three elements, colors, fabrics and elements; different combination among the rich elements will form different dress style, which will reflect different image model. Color is first senses presented to the audience so it has big influence on the first impression, thus the individual size, skin color and factors like this of the host group, combined with the stage lights, the audience's psychological feeling and finally the harmony of the other members of the host group, should be considered for choosing color. Diversity of apparel fabrics has a crucial role to create the costume with delicate style and color, which restricts the performance effect of clothing style. 
Apparel fabrics is the main carrier for discounting creative interpretation to the clothing of the designer; the fabric texture, color and luster, drapability and texture decide the creative direction of clothing, and the physical properties of the fabric more directly affect the wearing feeling. Style and decoration can shade the program positioning and make the partial decoration and the overall design fuse well at the same time, which plays an important role to enhance images stereo feeling and improve the image of the dress .Good clothing design elements can make the finishing point to image-building.

\section{The Difference and Unification between the Individual and Whole of the Host Group}

Host group is a special kind of host patterns, host group is a whole from the point of the program; host group as a whole, there must be differences between the individual from the point of the individual, thus it is one of the biggest challenges facing by host group is how to find the balance point of the costume design and the image-building between the diversity and unity. Make a research on the domestic host group show like "the Tianjin Night Carnival" of Tianjin TV, "Love Singing will Wins" of Zhengjiang TV, "Rural World" of 7 channel of CCTV and so on, there are only a few of them can create special features, thus it is easy to know that there are both advantages and potential dangers in host group. Such as the host group of all the previous Spring Festival gala on CCTV gathering the best host of CCTV, each host is a higher-ups in their own fields, but it does not prevent the mutual fusion, which brings the aesthetic enjoyment to the audience from the aspects of collocation of men and women, the transition of the shows and the clothing design. The most characteristic show is the one "Make Progress Everyday" of Hunan TV, The knowledgeable Han Wang is slightly older among the five hosts, Odie is from Taiwan who has a strong wacky imitation ability, Yuantian is graduated from the hosting major in university who is very stable, Feng Qian is delicate and fresh, Xiaowu is from Korea, each have their own characteristics in the clothing, in order to combine with the subject of the program, the costume of each host both includes their personality and builds the whole image of the " Everyday Bothers".

\section{PROFOUnd INFluence OF Costume Design to IMAGE-BUILDING OF THE HOST GROUP}

The image-building of the host group and the show style act on each other, as the packaged public image, the host group is the label of TV shows, whose costume design is the basis for image-building, supplements the image-building and is the first factor to serve the image-building. People perceive something through sight, hearing and other senses, and then get the overall impression via the abstract thinking, and the image is not the thing itself, but is the comprehensive representation of consciousness. So to raise the level of apparel design has a great impact to the brand of the image of host group, the influence to the brand of the image of host group caused by costume design has the following two points:

\section{A. Build the Inimitable Symbol to the Special Brand of Host Group, Improve the Brand Value of the Image of Host Group}

Brand value refers to the special meaning produced by the recognized brand by audience, it is a kind of high loyalty; at present, more and more TV programs realize the significance of brand value. The host group of shows build the brand value by using good clothing design, strengthen the connection with the audience in time and space; it is a style of representation which integrates the thematic, expressive and transmitted factors of the show; especially under the present penetration of all kinds of new media, the media nature of the host is unprecedented important to strengthen the brand value of the TV show. The function of "Happy Family" for set up the brand of Hunan satellite TV is visible, the five hosts work as a good team to pass a kind of family warm, bring this image to the show and TV silently and then pass it to the audience to meet the pursuit of the family emotion of them, so as to improve the loyalty to the overall brand of the host group. In a word, to improve the costume design level of the host group, to set up the good brand value of the host group and to build the clear threedimensional identification system can effectively take good advantage of the brand value of host group and further promote the brand value of the media.

\section{B. The Positive Image of Host Group Can Guide the Right Public Value}

In well-developed economy time, the aesthetic is no longer the elite culture but become a mass consumer goods, it is the low threshold for spreading promotes the aesthetic level. As a public figure, the host group will affect the public aesthetic silently, host group image is the guider for positive energy built by of TV programs, which should be full of responsible sense to the life of people whether in the programs or life out of the program. The host group of the brand show has very high value to the show itself both in little part of in word and deed and in big part of the clothing aesthetic, and even is the synonym of the show to some extent. Such as if Kangyong Cai is mentioned, then the show "Kangxi Come" will be associated; if the Han Wang is mentioned, then the "Make Progress Everyday" will be associated; if the jingtao is mentioned, the "University Student" will be associated and so on, so in the transmission process of TV, the optimistic and upward clothing aesthetic value and social value should be vigorously promoted to guide the right value orientation of public opinion. From the aesthetic point of view, the spread of the dress design of host group of TV show is infected by the popular culture, but the contained style beauty can be higher than life; the availability and flexibility of the content of TV media is more likely to affect the mass affected, the aesthetic ability is much easier to be promoted, through which to form a positive and healthy value orientation for the audience. 


\section{REFERENCES}

[1] Jihong Yang . Introduction to the requirement of sound-building and image-building of the host for radio show host $[\mathrm{J}]$. Journal of Scientific Research. 2015.3:286-286.

[2] Xiaolin Xiao. Brand-building of the TV host [J]. Modern communications: Journal of Beijing Radio and Television. 2015. (3) : 130-131.

[3] Lu Sun. Reset the role of the host of TV variety show under new situation[J]. Journal of Western Radio and Television. 2015 (8) : 6869.

[4] Juan Wen. Introduction to the diversity of sources for host of TV entertainment show [J]. Journal of Huaihai Institute of Technology: Humanities and Social Science Edition. 2015.13 (6) : 72-74.

[5] Hongyan Guo. Program host should find the right position at on-site interview [J]. China Prefecture and Tow and Newspaperman 2015 (6) : 99-100. 